\title{
Aufstiegskarrieren in der Schweiz
}

\author{
Felix Bühlmann*
}

\section{$1 \quad$ Einleitung}

Mit dem Aufkommen ausdifferenzierter Grossunternehmen in der ersten Hälfte des 20. Jahrhunderts und dem Ausbau des Bildungssystems verbreitete sich auch in der Schweiz der Traum vom sozialen Aufstieg mittels Aufstiegskarriere - vorwiegend bei Männern der unteren Mittelschicht (König et al., 1985). Die Aufstiegskarriere galt lange als besonders schlagendes, weil praktisches Argument der liberalen politischen Utopie einer kapitalistischen Bewältigung sozialer Ungleichheit. Ein möglichst hoher Anteil von sozial aufsteigenden bzw. absteigenden Biographieverläufen trüge zu einer sich stetig durchmischenden, ausgeglichenen und in ihrer Hierarchisierung besser legitimierten Gesellschaft bei (Lipset und Bendix, 1959). Sozialer Aufstieg sei dem Abbau der auf Ungleichheiten basierenden Spannungen zwischen den Klassen förderlich und streue Sand in das Getriebe politischen Klassenaktivismus. Bis heute ist daher sozialer Aufstieg von unbestrittener politischer und sozialer Brisanz. Die Erforschung sozialer Mobilität ist seit ihren Anfängen von quantitativen Ansätzen und strukturellen Erklärungen dominiert (Blau und Duncan, 1967; Erikson und Goldthorpe, 1992; Joye et al., 2003). Diese Forschungstradition hat viel zur Erforschung von absoluten bzw. relativen Mobilitätsraten, deren internationalem Spektrum und zur Ergründung von Ursachen von Mobilität und Reproduktion beigetragen. Gleichzeitig fiel es den traditionellen Ansätzen aufgrund ihres methodischen Instrumentariums schwer, soziale Aufstiege als biographische Verläufe zu fassen, das Zusammenspiel von Opportunitätsstrukturen und Motivlagen empirisch zu begreifen und den «subjektiven Aspekt» offen und biographisch situiert zu beschreiben. Dies gilt auch für den Fall der Schweiz: Spätestens seit der bahnbrechenden Studie von Levy et al. (1997) verfügen wir zwar über hochwertige quantitative Studien zur Mobilität, gebrechen aber an Wissen darüber, wie Aufstiege biographisch wahrgenommen werden, welche Mechanismen ihnen zugrunde liegen und welche Repräsentationen für die Akteure selber Handlungsrelevanz besitzen. Im vorliegenden Beitrag versuche ich daher den quantitativen Ansatz durch einen qualitativen zu ergänzen. Biographische Erzählungen schmiegen sich der Sequenzialität von Berufsverläufen an, sie erlauben Rückschlüsse auf das Zusammenspiel von Strukturen und Motiven und sie offenbaren, welche Repräsentationen für die Akteure bedeutsam sind. Ziel des vorliegenden Artikels ist es, aufzuzeigen wie die 
interaktionistische Karriere- und Biographieforschung zu einem besseren Verständnis von sozialer Mobilität - insbesondere von Aufstiegsmobilität - führen kann (Becker und Strauss, 1956).

Der Aufsatz ist wie folgt gegliedert: Im anschliessenden theoretischen Teil werden die relevanten quantitativen Ansätze der Mobilitätssoziologie diskutiert und deren Widerhall in der schweizerischen Mobilitätsforschung dokumentiert. Danach schlage ich eine in der interaktionistischen Karriereforschung verwurzelte Ergänzung vor und diskutiere die Forschungsstrategie sowie die verwendeten Methoden. Im empirischen Teil präsentiere ich vier Phasen der Aufstiegskarriere, zeige wie sie zeitlich strukturiert sind und wie sie von den Betroffenen gedeutet werden. Eine resümierende Diskussion der Befunde rundet den Artikel ab.

\section{Die Erforschung sozialer (Aufstiegs-)Mobilität}

\subsection{Mobilitätsforschung: Raten, Faktoren und Strukturen}

Die amerikanische Mobilitätssoziologie wurde in den 1950er Jahren durch Lipset und Bendix (1959) initiiert und darauf lange von der Idee des "Status Attainment» (Statuserwerb) von Blau und Duncan (1967) dominiert. Lipset und Bendix interessierten sich für Mobilitätsraten und beabsichtigen die Offenheit einer Gesellschaft zu messen, indem sie «reine Mobilität» von «struktureller Mobilität» unterschieden (Lipset und Bendix, 1959). Blau und Duncan (1967) untersuchten mit den von ihnen verfochtenen Statuserwerbsmodellen, welchen Einfluss beispielsweise der Status des Vaters oder der höchste erreichte Bildungstitel auf das Erreichen eines bestimmten Berufsstatus haben und wie Zusatzvariablen wie Rasse oder Geschlecht den Einfluss der sozialen Herkunft modulieren. Sie definierten Karrieren als graduell-hierarchische Berufs- oder Positionswechsel, die durch individuelles Erfolgsstreben, dem Streben nach «achievement», ausgelöst werden. Dieses simple Modell von Mobilität und Karriere erlaubte ihnen, eine grosse Anzahl Individuen mittels nationaler Umfragen zu untersuchen.

Spätestens Mitte der 1970er Jahre sah sich die Statuserwerbs-Theorie von strukturellen Theorien sozialer Schichtung und Mobilität herausgefordert. Die Vertreter dieser Theorien gruppierten sich um drei konzeptuelle Zugänge (Caroll und Mayer, 1986): Die Klassentheoretiker richteten ihren Fokus auf die Formierung demographisch und soziokulturell homogener Klassen qua Mobilitätsprozesse (Erikson und Goldthorpe, 1992). Die Arbeitsmarktsoziologen beobachteten wie eine Zweiteilung des Arbeitsmarktes sozialen Aufstieg kanalisiert (Doeringer und Piore, 1971). Die Organisationstheoretiker schliesslich plädierten dafür, die mikround makrosoziologischen Aspekte mittels Erforschung einzelner Organisationen zu verbinden (Spilerman, 1977; Baron und Bielby, 1980, 738). Auch wenn sich einzelne Organisationsforscher durchaus für Karrieresequenzen interessierten, un- 
terschieden sich die Methoden und Forschungsstrategien der strukturellen Theorien nicht grundsätzlich von den Statuserwerbsmodellen.

\subsection{Schweizerische Echos in den 1990er Jahren}

Das Operieren mit Raten, Faktoren und Strukturen gehört auch in der Schweizer Mobilitätssoziologie zum Kanon und dominiert eine Mehrheit der einschlägigen, sich fast ausnahmslos an amerikanischen Vorbildern orientierenden Studien. Was die sozialwissenschaftliche Forschung zur Mobilität in der Schweiz lange von derjenigen in den USA oder Grossbritannien unterschied, waren nicht die Konzepte oder die Methoden, sondern die fehlenden landesweiten Datensätze. Obwohl der Topos schon früh debattiert und erforscht wurde (siehe zum Beispiel Girod, 1977), litt die schweizerische Mobilitätsforschung an ihrer regionalen bzw. zum Teil auch thematischen Beschränktheit (Levy und Suter, 2002). Dies änderte sich in den frühen 1990 er Jahren mit der breit angelegten Untersuchung von Levy et al. (1997) und mit der sukzessiven Teilnahme der Schweiz an internationalen Studien, wie zum Beispiel dem International Social Survey Program. Die neue Datensituation schob eine neue Welle schweizerischer Mobilitätsstudien an, welche einerseits von der Universität Zürich (Stamm et al., 2003), andererseits von den Universitäten Lausanne und Neuenburg (Levy et al., 1997) ausgingen. Diese Untersuchungen der späten 1990 er und frühen 2000 er Jahren konnten endlich auf eine solide nationale Datenbasis bauen, die es erlaubte, die Entwicklungen in der Schweiz im internationalen Kontext zu situieren. In ihrer Studie interessierten sich Levy et al. für die soziale Mobilität als einen essentiellen Aspekt der schweizerischen Sozialstruktur (Joye und Levy, 1994; Levy et al., 1997). Diesen Ansatz verfeinernd, untersuchten Bergman und Joye die Veränderungen der Mobilitätsmuster im Laufe der krisenhaften 1990er Jahre (Bergman et al., 2002) und warfen einen methodisch ausgefeilten Blick auf die Bildungsmobilität zwischen 1975 und 1990 (Joye et al., 2003). Ein Stiefkind sowohl der angloamerikanischen als auch der schweizerischen Mobilitätsforschung blieb hingegen der «subjektive Aspekt» sozialer Mobilität. Nicht dass er gänzlich abwesend gewesen wäre: in der Studie von Levy et al. (1997, 495-538) wurde ihm ein ganzes Kapitel gewidmet; und auch Stamm et al. (2003, 125-141) befassen sich explizit mit dem Verhältnis von Strukturen und Repräsentationen.

\section{Biographie- und Karriereforschung als Alternative}

\subsection{Die interaktionistische Karrieresoziologie}

Um die soziale (Aufstiegs-)Mobilität aus einer alternativen Perspektive zu beleuchten und den Blick auf diesen subjektiven Aspekt sozialen Aufstiegs zu schärfen, bietet es sich an, die Erforschung von Aufstiegskarrieren mit einem auf biographischen 
Interviews basierenden Zugang zu ergänzen. ${ }^{1}$ Die qualitative Sozialforschung kann mit dem symbolischen Interaktionismus direkt auf einen Theoriestrang zurückgreifen, der über ein eigenes Karrierekonzept verfügt und dieses immer in sequenziellen Begriffen gefasst hat.

Als begrifflicher Nachfolger des "Life-History-Konzepts» wurde das Karrierekonzept von Hughes und seinen Schülern im Rahmen ihrer Berufs- und Professionssoziologie entwickelt (Hughes, 1937; Becker und Strauss, 1956). Der am lebendigsten erinnerliche Beitrag von Hughes ist seine Unterscheidung zwischen der «objektiven» und der «subjektiven» Karriere. Während die erstere sich auf eine Sequenz von sozialen Positionen bezieht, so umfasst letztere auch die Abfolge der sich im Lebensverlauf verändernden subjektiven Deutungen und Identitäten (Hughes, 1937, 413). Bis in die 1970er Jahren wurde die Tradition in erster Linie von Hughes' US-Amerikanischen Schülern weitergetragen, die - funktionalistische Ansätze kritisierend - das Karrierekonzept für allgemein verlaufsförmige Phänomene wie Drogenkarrieren, Krankheitsverläufe oder Heimkarrieren öffneten. Aufzeigend wie spezifische Institutionen («Szenen», Spitäler oder Psychiatrische Anstalten) mit den subjektiven Deutungsmustern der Akteure zusammenspielten, beleuchteten die Hughes-Schüler insbesondere deviante, diskontinuierliche, absteigende oder horizontale Karriereformen - welche von den auf die männlichen Aufstiegskarrieren fixierten Mobilitätssoziologen gerade aussen vor gelassen wurden (Barley, 1989). Ende der 1970er Jahre überquerte die interaktionistische Biographiesoziologie den Nordatlantik: Während Bertaux $(1981,2005)$ den Begriff Life-History als «récit de vie» übersetzte, prägte Fritz Schütze (1983) den Begriff «Verlaufskurve» für den deutschen Sprachraum. Standen die beiden Traditionen zu Beginn der 1980er Jahren noch im regen Austausch (Bertaux, 1981), so haben sich mittlerweile zwei relativ eigenständige nationale Forschungsräume herausgebildet. Diese widmeten sich in erster Linie der methodologischen Verfeinerung und kamen dabei zu weitgehend übereinstimmenden Erkenntnissen (Schütze, 1983; Rosenthal, 1995; Demazière und Dubar, 1997).

\subsection{Die Vorzüge des Karrierekonzepts}

Das ursprüngliche Karrierekonzept von Hughes findet, von den erwähnten methodischen Neuerungen kaum modifiziert, noch immer rege Verwendung (WohlrabSahr, 1995; Demazière und Dubar, 1997). Drei Stärken machen es zur Analyse von Aufstiegskarrieren besonders fruchtbar:

Erstens konzipiert der Karrierebegriff soziale Phänomene per definitionem als verlaufsförmig. Misstrauisch gegenüber statischen Erklärungen behauptete Hughes, dass soziale Identitäten kontinuierlich revidiert und den Ereignissen des objektiven Karriereverlaufs angepasst würden (Hughes, 1937, 410). Deshalb geht die biogra-

1 Diese Kombination ist in der Schweiz noch kaum verbreitet. Ausnahme bildet bis heute Schmeisers Artikel zu sozialen Abstiegen in Akademikerfamilien (Schmeiser, 2000). 
phische Forschung davon aus, dass es, um ein Phänomen zu durchdringen, in erster Linie einer Rekonstruktion seines prozesshaftes Entstehens bedarf (Demazière und Dubar, 1997). Dieses Vorgehen erlaubt, Zeitstrukturen und Übergänge als elastische, manchmal fliessende Prozesse zu verstehen - ohne deren Verlauf in konzeptuell rigide, gegeneinander abgetrennte Zeitsegmente zwängen zu müssen (Becker und Strauss, 1956). Zudem setzten die Karriereforscher Vergangenheit, Gegenwart und Zukunft zueinander in Beziehung: in der Gegenwart gemachte Aussagen sind laut ihnen nur zu fassen, wenn man sie als Resultat des bisherigen Biographieverlaufs und als Verweis auf den für die Zukunft ausgemalten Weg begreift (Fischer und Kohli, 1987; De Coninck und Godard, 1989; Rosenthal, 1995).

Zweitens entspringen aus der Perspektive der symbolischen Interaktionisten Karrieren dem Zusammenspiel von individuellen Motiven und sozialen Strukturen (Becker und Strauss, 1956). Durch die Unterscheidung zwischen objektiven und subjektiven Karrieren, waren sie in der Lage beide Aspekte theoretisch aufeinander zu beziehen. Sie übersetzten ihre Erkenntnisse in empirische Strategien und generierten über offene, biographische Interviews zugleich Erzählungen über die Abfolge der institutionellen Positionen und die Wahrnehmungen, Reaktionen und Handlungen der Akteure (Rosenthal, 1995). In der Rekonstruktion schliesslich können diese beiden Aspekte erklärend zu einander in Beziehung gesetzt und für die Theoriebildung urbar gemacht werden.

Drittens führt die grössere Offenheit biographischer Interviews dazu, dass ein breiterer Fächer von Repräsentationen zur Sprache kommt, den relevanten Repräsentationen besonderes Gewicht verliehen wird und diese Deutungsmuster biographisch situiert werden (Schütze, 1983). Spezielle theoretische Aufmerksamkeit genoss in der interaktionistischen Tradition die Verknüpfung von Karriereverläufen mit sozialen Identitäten (Hughes, 1937). Ihre Vertreter erklären Identitäten als ein Produkt von beruflichen Karrierebewegungen und interessieren sich dafür, wie die entstandenen Identitäten wiederum an der Ausgestaltung organisatorischen Strukturen teilhaben (Becker und Strauss, 1956).

\section{Forschungsstrategien}

Diese Vorzüge der interaktionistischen Karriereforschung nutzend, versuche ich im Folgenden nachzuzeichnen, in welche Phasen soziale Aufsteiger ihre Karrieren einteilen, mit welchen Zeitstrukturen sie diese Phasen punktuieren und welche Bedeutungen sie ihnen zuschreiben. Methodisch berufe ich mich dabei auf die Weiterentwicklungen des interaktionistischen Ansatzes, insbesondere französischer und deutscher Provenienz (Schütze, 1983; Fischer und Kohli, 1987; De Coninck und Godard, 1989; Rosenthal, 1995; Wohlrab-Sahr, 1995; Demazière und Dubar, 1997; Bertaux, 2005). 


\subsection{Stichprobe}

Um Aufstiegskarrieren in der Schweiz zu verstehen, untersuche ich eine Gruppe von Akteuren, die zu Beginn ihres Berufsverlaufes den Wunsch äussern, mittels einer Karriere sozial aufzusteigen. Weil gewisse Professionen strukturell an eine relativ enge Auswahl von möglichen Karriereverläufen gebunden sind, ist es für die Selektion von Akteuren mit Aufstiegswunsch besonders aussichtsreich, typische Aufstiegsprofessionen in den Blick zu nehmen. Dieses Programm wurde umgesetzt, indem zwei Berufe mit einer Tendenz zur aufsteigenden Mobilität ausgewählt wurden: Ingenieure mit Ausbildung an einer Höheren Technischen Lehranstalt (HTL) und Betriebswirtschafter mit einem Titel einer Höheren Schule für Wirtschaft und Verwaltung (HWV). Beides sind numerisch und symbolisch von Männern dominierte Berufe: Der erste eine Stütze der Schweizer Industrie, letzterer ein Generalistenberuf im Servicesektor. Zudem können in der Schweiz beide Berufe an «höheren Fachschulen" gelernt werden. ${ }^{2}$ Für diesen Bildungsweg absolvieren die Akteure zuerst eine Lehre und besuchen dann, meist nach einigen Jahren Berufsausübung, eine höhere Fachschule. Mit dem an der höheren Fachschule erworbenen Titel hoffen sie ihre Chancen auf einen sozialen Aufstieg zu erhöhen. Die Entscheidung eine höhere Fachschule zu besuchen, kann deshalb als eine praktische Bestätigung der Aufstiegsambitionen gewertet werden.

Die folgende Analyse beruht auf 28 qualitativen biographischen Interviews mit einer Stichprobe von männlichen HTL-Ingenieuren und HWV-Betriebsökonomen, durchgeführt im Jahr $2005^{3}$ (vgl. Tabelle 1). Die Auswahl der Interviewpartner wurde von einer Typologie angeleitet, die über eine Optimal Matching Analyse ermittelt wurde (Bühlmann, 2008). Aus jedem der mit diesem Verfahren identifizierten sechs Typen wurden vier bis sechs Akteure ausgewählt und zusätzlich nach Alter und wirtschaftlicher Branche variiert. ${ }^{4}$ Solch eine Auswahl per Stichprobenplan dient dazu, eine möglichst breite Varianz von Karriereverläufen abzudecken (Kelle und Kluge, 1999). Sie gilt als Alternative gegenüber ausschliesslich auf qualitativen Methoden beruhenden Strategien der Maximierung und Minimierung der Kontraste (Strauss und Corbin, 1996). ${ }^{5}$

2 Sämtliche Interviewpartner sind vor 1975 geboren (s. Tabelle 1) und haben die höheren Fachschulen noch vor den Reformen der 1990er Jahre besucht, aus denen das heutige Fachhochschulsystem hervorging.

3 Ich konzentriere mich in diesem Beitrag auf Aufstiegskarrieren als einen männlichen Berufsverlauf. Ein Vergleich zwischen männlichen und weiblichen Karrieren kann an dieser Stelle nicht geleistet werden.

4 Lediglich die Erzählungen der Aufstiegswilligen wurden erfragt. Aus Zeitgründen war es nicht möglich, parallele Interviews mit dem Umfeld der Interviewten (Familie, Freunde) zu führen, um ein facettenreicheres Bild ihrer Karrieren zu erhalten.

5 Wenn auch die beiden Methoden nicht zu einer grundsätzlich anderen Auswahl führen, so besteht der Vorteil von Optimal Matching darin, dass eine grosse Anzahl von längeren Karrieresequenzen miteinander verglichen werden können, deren Muster sich auch dem geübten Auge nicht immer unmittelbar erschliessen. 
Tabelle 1 Anzahl befragter Personen nach Geburtskohorte und Profession

\begin{tabular}{lccc}
\hline & Ingenieure & Betriebswirtschafter & Total \\
\hline-1955 & 9 & 2 & 11 \\
$1956-1965$ & 6 & 5 & 11 \\
$1966-1975$ & 2 & 4 & 6 \\
Total & 17 & 11 & 28 \\
\hline
\end{tabular}

\subsection{Biographische Interviews}

Die Interviews wurden als offene biographische Gespräche geführt (Schütze, 1983). $\mathrm{Zu}$ deren Beginn wurden die Interviewten aufgefordert, ihre (Berufs-) Biographie zu erzählen. Die darauf folgende Erzählung wurde wenn möglich nicht unterbrochen. Lediglich in späteren Phasen des Interviews ermunterte ich die Befragten spezifische Erzählstränge, thematische Blöcke oder biographische Phasen in narrativer Form wiederaufzunehmen (familiärer Hintergrund, Ausbildung, Berufsverlauf, Familie und Zukunft) - wenn diese bis zu diesem Zeitpunkt unerwähnt oder an der Oberfläche geblieben waren. In einer dritten Phase erfragte ich offengebliebene oder unklare Sachverhalte und stellte zu wichtig erscheinenden Ereignissen und Entscheidungen Warum-Fragen (Schütze, 1983, 285). Die Interviews dauerten im Durchschnitt 75 Minuten und wurden anschliessend vollständig transkribiert und mit Atlas-ti aufbereitet. Bei diesen Interviews handelt es sich um retrospektive Erzählungen der eigenen Berufslaufbahn - basierend auf aus der Gegenwartsperspektive hervorgehenden Selektionen, Gewichtungen und Interpretationen der Ereignisse (Rosenthal, 1995). Aus den Erzählungen lassen sich sowohl der durchlebte Karriereverlauf (mit einer Abfolge von Ereignissen, Wendpunkten und Phasen) ablesen, als auch die biographisch entstandenen Wahrnehmungskategorien, Motivlagen, Denkmuster und Interpretationen der Akteure extrahieren, welche die vergangenen, aktuellen und zukünftigen Handlungen der Aufstiegswilligen anleiten (Rosenthal, 1995). Die Rekonstruktion von Biographieverläufen setzt diese zwei Ebenen zueinander in Beziehung und generiert aus dem so geschaffenen doppelten Referenzrahmen theoretische Erklärungen zum Biographieverlauf (Schütze, 1983; Strauss und Corbin, 1996)

Schützes Analyseverfahren leicht vereinfachend, unterscheide ich drei Analyseschritte (Schütze, 1983): In einem ersten Schritt rekonstruiere ich den erlebten Biographieverlauf. Es sollen also die wichtigsten Phasen wie Schule, Berufsbildung, berufliche Positionen, Perioden der Arbeitslosigkeit und die sie verbindenden Ereignisse und Übergänge wie Bildungsabschlüsse, Anstellungen, Promotionen, Degradierungen, Entlassungen oder Pensionierungen als «faktische Prozessabläufe» herausgearbeitet werden (Schütze, 1983, 286). Zweitens rekonstruiere ich wie die Befragten ihren Biographieverlauf deuten, definieren und legitimieren. Zentral sind dabei die zeitliche Strukturierung der Karriere, die Bedeutung der Übergänge und die Einteilung in spezifische Phasen - die die Befragten meist im Zusammenhang 
mit kulturell (und insbesondere milieuspezifisch) vorgegebenen Mustern referieren. Dazu gehören ihre subjektiven Wahrnehmungen der strukturellen Optionen, ihre Vorstellungen von Normalität, ihre Überlegungen, Entscheidungen und deren Legitimation (Schütze, 1983; Demazière und Dubar, 1997). Drittens versuche ich aus einer Gegenüberstellung des tatsächlich Erlebten und des Erzählten eine Theorie des sozialen Aufstiegs zu konstruieren und diese mittels kontrastiven Vergleichen zwischen einzelnen Fällen zu abstrakteren Schemata zusammenzufassen, ${ }^{6}$ bzw. sie dort mit Varianten zu ergänzen, wo sie nicht auf einen einzigen Nenner gebracht werden konnten (Schütze, 1983, 287). Um den Akzent auf die Gemeinsamkeiten der Interviewten und die strukturellen Regelmässigkeiten ihrer Narrationen zu legen, entschied ich mich, die Interviews synoptisch und nicht in Form von Fallrekonstruktionen darzustellen (Kelle und Kluge, 1999).

\section{Aufstiege im Spiegel biographischer Erzählungen}

Im Allgemeinen erzählen die Ingenieure und Betriebswirtschafter ihre Karriere in vier verschiedenen Phasen. Diese nenne ich im Folgenden die Phase des Aufwachens (5.1), die Mauserungsphase (5.2), die Versuchsphase (5.3) sowie die Aufstiegs- und Konsolidierungsphase (5.4).

\subsection{Aufwachen als individueller Ausbruch}

Mit der "Aufwachphase» bezeichne ich den Moment in dem die Interviewten realisieren, dass sie einen weiteren sozialen Aufstieg anstreben. Man könnte ihre Aspirationen zunächst als etwas einschätzen, dass sie recht unvermittelt «entdecken». Die Befragten sagen beispielsweise, sie hätten zu einem bestimmten Zeitpunkt «realisiert», dass sie noch "weiter kommen» möchten oder noch etwas «mehr erreichen» wollen. Spiegelbildlich sprechen andere von einem Moment, wo sie sich nicht mehr vorstellen konnten, «die nächsten 40 Jahre hinter diesem Schalter zu verbringen». Am akkuratesten begreift man die zeitliche Entstehungsstruktur des Aufwachens als einen langsamen Konkretisierungs- und Explizierungsprozess. Das Streben weiter zu kommen äussert sich anfangs als vage und unaussprechbare Intuition und wandelt sich langsam zu einem klaren und schliesslich verbalisierbaren Wunsch. Bevor der Wunsch «weiter zu kommen» formuliert und konkret angekündigt wird, flackert er periodisch auf, wenn sich die Möglichkeit dazu bietet. Implizite Anzeichen dafür sind zum Beispiel ein Flirt mit dem Gymnasium oder die Wahl einer besonders herausfordernden, modernen und verheissungsvollen Lehre. ${ }^{7}$ Meist während der

6 Es ist freilich zu beachten, dass die Befragten zu verschiedenen Momenten ihrer Biographie befragt wurden. Die Verläufe der jüngeren Kohorten sind inkomplett. Aus diesem Grund ist es nicht möglich die späteren Karrierephasen im Kohortenvergleich zu beschreiben.

7 Zeichnerische Berufe, Feinmechaniker, TV- und Radioelektriker in den 1960er und 1970er Jahren und Elektroniker und Informatik-Berufe in den 1980er und 1990er Jahren. Im Dienst- 
Lehre lassen die Karrierekandidaten diesen Vorankündigungen einer in ihnen schlummernden Disposition eine mehr oder weniger offene Erklärung an die soziale Umgebung folgen. Eine Minderheit wagt ihre Träume vom sozialen Aufstieg erst nach ein paar Jahren beruflicher Praxis zu realisieren, meist unter Einfluss negativer Erlebnisse oder angesichts von Ängsten davor, in einer herausforderungsarmen Funktion zu «versauern».

Die zeitliche Struktur des Aufwachens ist nur auf der Folie der für die Befragten typischen milieuspezifischen Konzeption von biographischer Normalität verständlich. Die Mehrheit der künftigen Ingenieure und Betriebswirtschafter stammt aus Familien, in denen die Eltern selber eine Lehre gemacht und als qualifizierte Handwerker bzw. Angestellte gearbeitet haben. Die verbreitete Antwort auf die Frage, wie sie ihre Jugendzeit erfahren hätten, lautet, sie hätten eine «normale» Schulzeit erlebt.

\section{Ich habe normal Volksschule besucht, sechs Jahre Primarschule und nach- her drei Jahre Sek. Die Berufswahl ist bei mir eigentlich fast eher ein bisschen zufällig gewesen, ich bin nie in einer Berufsberatung gewesen. Ich habe gewisse Vorstellungen gehabt von dem was ich lernen möchte, für mich ist eigentlich klar gewesen ein handwerklicher Beruf. \\ (Ingenieur, ${ }^{*} 1957$ )}

Für die meisten Ingenieure und Betriebswirtschafter bedeutet «normal» in direkter Abfolge die Primarschule und die Sekundarschule zu besuchen und im Anschluss daran eine Lehre im handwerklichen oder im kaufmännischen Bereich in Angriff zu nehmen. Als Gegensatz dazu codieren sie den Besuch der Realschule oder des Gymnasiums als «nicht-normal». Die «normale» Zwischenposition grenzt die Akteure einerseits gegen Kinder von Migranten und unqualifizierten Arbeitern und andererseits gegen mit Bildungskapital dotierte Gymnasiasten mit Universitätsambitionen ab. Die Wahl der Lehre ist für sie sozial selbstverständlich und basiert auf einem impliziten Konsens zwischen dem Akteur, seinen Eltern und seiner sozialen Entourage.

Im Gegensatz zu diesem "normalen» Ablauf wird die Entscheidung, die höhere Fachschule zu besuchen, als ein individueller Ausbruch erzählt. Die Befragten belieben zu unterstreichen, dass diese Entscheidung ihre ganz persönliche war, dass sie von ihren Eltern weder dazu gedrängt noch dabei unterstützt worden seien. In einigen Fällen präsentieren sie die Entscheidung sogar als eine Wahl gegen die Eltern, die ihnen von Personen ausserhalb ihres angestammten Milieus angetragen wurde. Ein Ingenieur kleidet die Erzählung seines Ausbruchs in folgende Worte:

Ich bin der Älteste gewesen von diesen fünf und darum eh, ja habe den Weg selber machen müssen, habe keine Vorbilder ... es hat auch in der

leistungsbereich blieb unverändert die kaufmännische Lehre das generalistische Sprungbrett für eine künftige Karriere. Hier steht insbesondere die «Bankenlehre» für höhere Ambitionen. 
Umgebung, also ich wüsste niemanden der dort Ingenieur oder so etwas gehabt hat, das ist mein eigener Weg gewesen, ich habe gesehen einfach "Mathi, Geometrie, Zeichnen" und diese Kombination, da wird irgend einmal etwas daraus werden. (Ingenieur, *1955)

Die Aufwachphase der Ingenieure und Betriebswirtschafter kann also in ihrer Strukturierung als doppelte Entscheidung verstanden werden. Die milieukonforme Entscheidung für eine solide Lehre wird ergänzt, durch einen individuellen, gegen das elterliche Umfeld und ins Unbekannte gerichteten Ausbruch.

Die Erzählungen über den Ausbruch aus der Normalität können auf drei Varianten kondensiert werden. Eine erste Gruppe präsentiert sich als von ausserfamilialen Vorbildern inspiriert. Künftige Ingenieure, typischerweise jene, die ihre Lehre in kleinen Planungsbüros absolvieren, machen ihr Erwachen narrativ an der Begegnung mit der Figur des unabhängigen und schöpferischen Ingenieurs fest, der «interessante Probleme» mit Hilfe «komplexer Technologie» löst. Dieser Mentor informiert den Lehrling ganz praktisch über den Raum des Möglichen: beispielsweise indem er ihn über die Atmosphäre, die Perspektiven oder den vermittelten Stoff an den höheren Fachschulen aufklärt. Der Ausbruch kann aber auch über repulsive Begegnungen in Gang gesetzt werden. Typischerweise durch die Figur des körperlich verbrauchten älteren Handwerkers, als Negativ-Vision ihrer eigenen Zukunft. Andere zitieren die primitiven und rohen Gebräuche im Universum der Handwerker, die sie zunehmend abgestossen hätten. ${ }^{8}$

Eine zweite Gruppe von Ingenieuren und Betriebswirtschaftern berichtet, dass ihre Entscheidung an die höhere Fachschule zu wechseln, durch von ihnen als objektiv empfundene, ihnen ein gewisses Potential attestierende «Beweise» befördert worden sei. Dabei kann es sich um von einem Vorgesetzten im informellen Gespräch bescheinigte Kompetenzen wie «eine schnelle Auffassungsgabe» handeln; aber auch um "gute Noten» in der Berufsschule, die als eine bis zu einem gewissen Grad neutrale, milieufremde Instanz über die Leistungsfähigkeit der Lehrlinge urteilt.

Ich habe schon gedacht, irgend etwas mache ich dann früher oder später noch, mit einem 5.7, einfach jahrein, jahraus nur arbeiten, dreckige Hände und so ... es gibt Angenehmeres ... (Ingenieur, *1967)

Diesem Ingenieur gaben die guten Noten in der Berufsschule den Anlass dazu, von einer Überwindung seiner Situation zu träumen und der Routine und dem Dreck mit Hilfe einer weiteren Ausbildung mittelfristig zu entfliehen. Solche Bestätigungen benötigen die Interviewten als Legitimation für sich selber und ihrem sozialen Umfeld gegenüber, um Ambitionen und Pläne für die Zukunft zu entwickeln.

8 Kaum erstaunlich, dass dies «Arbeiterklassen-Figuren» sind, von denen sich die künftigen Ingenieure und Betriebswirtschafter mit ihrem Aufstieg abgrenzen möchten. Klagen über die dreckigen Sprüche und das primitive Benehmen sind oft zu hören und funktionieren dem Interviewer gegenüber auch als indirekte Selbstbeschreibung. 
Die Unzufriedenheit mit der aktuellen Situation bzw. mit der sich unter gegenwärtigen Bedingungen ausgemalten Zukunft bemüht eine dritte Gruppe, um dem Interviewer ihr damaliges Streben nach Höherem zu erläutern. Sie erzählen eine Geschichte der Bewusstwerdung einer Diskrepanz zwischen ihrer Realität und ihren Träumen. Ein solcher Graben scheint sich zeitlich langsam zu vertiefen - er wird kaum mit einem präzise bestimmbaren Ereignis in Verbindung gebracht.

Etwa 5 Jahre nach dem Ende der Lehre habe ich dann gesagt, so jetzt muss ich noch irgend etwas machen, es kann nicht sein, dass ich jetzt die nächsten 40 Jahre da jetzt kaufmännischer Angestellter bin der irgendwo ein bisschen schäfferle und versauern und einfach nicht weiterkommen ... (Betriebswirtschafter, ${ }^{*} 1973$ )

Der Ausschnitt illustriert wie die Frustration und die daran anschliessende Strategie die höhere Fachschule zu besuchen dem kontinuierlichen Vergleich zwischen herbeigesehnter und der unter den herrschenden Bedingungen als normal imaginierten Laufbahn entspringt. Frappierend: Karrieren werden als Vehikel dargestellt, um sich einer «langweiligen», "dumpfen» und «stagnierenden» Zukunft zu entziehen. Anstelle der üblicherweise als Brennstoffe von Karrieren präsentierten Träume, Pläne und Ambitionen handelt es sich hier um Frustrationen und Ängste, die die Akteure dazu veranlassen, Karrieren einzuschlagen.

\subsection{Bestätigung, Ablösung und neue Banden während der Mauserungsphase}

Im Anschluss an die Ankündigung ihrer Pläne, geht es darum, diese mit dem Besuch der höheren Fachschule praktisch umzusetzen. Die Bildungsphase an der höheren Fachschule liefert den zugleich praktischen und symbolischen Nachweis der Seriosität ihrer Aufstiegsträume. Bis zum Beginn der Schule, bleibt die Entscheidung eine vorläufige und umstossbare. Gelegenheiten sie zu verinnerlichen und gegenüber dem Umfeld zu bestätigen sind daher willkommen: folgt man den Erzählungen der Befragten, bieten sich den jungen Karriereaspiranten eine Reihe von Ritualen, um (1) ihre Ernsthaftigkeit unter Beweis zu stellen, (2) sich von ihrem Ursprungsmilieu moderat zu lösen und (3) Banden zu vergleichbar aufwärtsorientierten Kollegen zu knüpfen. Im Ergebnis nehmen sie über diese Vehikel langsam eine neue soziale Identität an und mausern sich gegenüber ihrem Herkunftsmilieu.

Zwei Institutionen eignen sich besonders gut dafür, sich über die Richtigkeit und Aufrichtigkeit der Entscheidung zu versichern: die Initiationsreise ins Ausland und die Militärkarriere als einstweiliges Surrogat und Übungsfeld der Wirtschaftskarriere. Mit Reisen oder Sprachaufenthalten im Ausland überbrücken die Befragten zeitliche Löcher just bevor oder nach der höheren Fachschule. Diese Kurzaufenthalte dienen latent dazu, so mein Schluss, sich seiner selbst zu versichern und dem sozialen Umfeld zu signalisieren, dass der Wunsch die höhere Fachschule zu besuchen 
mehr als ein Teenagertraum gewesen ist. Dann funktioniert diese Reise aber auch als Loslösung vom Elternhaus und als eine zusätzliche Lernphase - oft ist sie mit dem Besuch einer Sprachschule verknüpft. Die Aufenthalte funktionieren aber auch als Test seiner selbst. Bin ich eigenständig, schaffe ich es mich durchzuschlagen? Das Engagement in der Schweizer Armee gilt den Ingenieuren und Betriebswirtschaftern als zweite Möglichkeit, die Zeit vor oder nach der höheren Fachschule zu überbrücken. Hier habe sich ihnen die Chance geboten, ihre Fähigkeiten in der Praxis unter Beweis zu stellen und zu testen, ob sich ihr Potential zum Aufstieg diesem, der Wirtschaftswelt nicht unähnlichen Praxistest standhält. Ein grosser Teil der Befragten durchläuft denn auch die Unteroffiziersschule und interpretiert ihre Berücksichtigung bei der Beförderung als Bestätigung ihrer Fähigkeiten; die Führungsaufgabe nehmen sie als eine erste Anwendung ihrer Kapazitäten wahr, die sie auf Führungspositionen in der Privatwirtschaft zu übertragen gedenken. Rückblickend taxieren sie die Armee als eine willkommene und wiederum «objektive», da milieufremde Instanz, die ihre Ambitionen bestätigt und ihren Glauben an ihre Fähigkeiten zu festigen vermochte.

Die massgebliche Konkretisierung der Ambitionen allerdings ist das Studium an der höheren Fachschule selbst. Die Erzählungen der Befragten über das Studium sind gespickt mit Geschichten über das «lustige Studentenleben» Es wird geschildert, wie sie das Leben "genossen» haben, wie sie von «mannigfaltigen Freiheiten» profitierten, viele «neue Freunde» fanden und überhaupt die «beste Zeit ihres Lebens» hatten. Zugleich schleichen sich aber auch Anspielungen darauf ein, wie "hart» die Zeit gewesen sei: eine Menge Stoff, harte Arbeit und soziale Isolation. Besonders die Absolventen der Abendschule sehen die Ausbildung rückblickend auch als entbehrungsreiches Opfer. Sie habe einen Grossteil der Freizeit monopolisiert und sie von ihren Freunden getrennt. Symbolisch überschreiten die Befragten mit dem Besuch der höheren Fachschule auch Grenzen beruflicher Identität. Dies zeigt sich exemplarisch bei den Ingenieuren: Ihre handwerklichen Lehren waren mit Arbeit im Freien oder in der Werkstatt verbunden - Dreck, Gestank, Hitze, Wind und Kälte gehörten zu ihrem Alltag. Die Rückkehr ans Schulpult entspricht für sie deshalb auch einer Grenzüberschreitung Richtung «Büro» und «Kopf».

\section{Im Tagestech bin ich in den Ferien wieder auf diese Lastwagen arbeiten gegangen und dort muss ich sagen, dort ist es mir verleidet mit diesem Werkstattgeschmack. Weil die Lastwagen haben Diesel und das stinkt recht. Wenn dir der über das Übergwändli runterläuft dann stinkst du und einfach jeden Abend hast du duschen gehen müssen bevor du dich hast anschauen können. Da habe ich sagen müssen "nein das kann es nicht sein». (Ingenieur, 1967)}

Auch die künftigen Betriebswirtschafter situiert der Gang an die höhere Fachschule gegenüber ihren alten Arbeitskollegen neu. Allerdings sind ihre zu überwindenden 
Mauern niedriger, die Landung sanfter: weder wechseln sie den Arbeitsplatz noch müssen sie mit grundsätzlich neuen Instrumenten und Techniken arbeiten lernen.

Im Vergleich zu den Schweizer Universitäten, deren Besuch den jungen Studierenden oft eine Wohnortsverlegung abverlangt, ist das Netz der höheren Fachschulen enger gewoben, ihre Profile und ihr Fächerangebot recht ähnlich. Ihr Besuch bedingt deshalb nicht im selben Ausmass eine geographische Trennung vom Elternhaus. Trotzdem entflieht ein beträchtlicher Teil der angehenden Betriebswirtschafter und Ingenieure dem direkten Einfluss ihrer Familie und Freunde. Die Trennung von ihren alten Freunden beurteilen im Nachhinein insbesondere jene, die die Abendschule besuchten, als folgenreich. Die zeitaufwändigen Stundenpläne und der erhebliche Lernaufwand habe es ihnen kaum erlaubt, ihre alten Freunde weiterhin zu frequentieren. Ein Ingenieur schildert die Konsequenzen der Wiederaufnahme der Schule für sein soziales Netz in folgenden Worten:

Das hat sich dramatisch verändert, absolut. Nach dem Abschluss der Ingenieurschule habe ich komplett einen anderen Kollegenkreis gehabt. Von denen von vorher hat keiner überlebt, ich habe keine Kontakte mehr gehabt. Das bedingt, dass man ein komplett neues Beziehungsfeld aufbaut. (Ingenieur, *1957)

In den meisten Fällen setzt sich der neu erworbene Freundeskreis nicht zufällig zusammen, sondern umschliesst in erster Linie Mitstudenten. Diese stammen aus ähnlichen Verhältnissen, sind von einem vergleichbaren Wunsch aufzusteigen beseelt - sie haben ähnlich zukunftsorientierte und fordernde Einstellungen, Ideen und Träume.

An der HWV hat es viele Leute gehabt die gesagt haben "ok», die noch einen Hunger gehabt haben, eine Wissbegier. Von den Leuten her ist das angenehm gewesen, es hat nie jemanden gesagt "uhh, wir haben zu viele Aufgaben» oder so, sondern "ok, das gehört dazu, das machen wir».

(Betriebswirtschafter, ${ }^{*} 1971$ )

Typischerweise bedeuten solche neu geschlossenen Banden eine Abgrenzung und manchmal gar Ablehnung jener, die sich diesen Ambitionen verweigern. Interessanterweise sind die heutigen Freunde der Befragten oft noch die Klassenkameraden von damals - wir können also annehmen, dass das gemeinsame Durchschreiten dieser Loslösungs- und Neubindungsphase starke und lang anhaltende Beziehungen schafft.

\subsection{Die Versuchsphase und die Suche eines Karriereankers}

Die Versuchsphase beginnt mit der ersten Anstellung nach dem Abschluss der Fachhochschule und endet zum Zeitpunkt, wenn eine "angemessene» Stelle gefunden wird, 
die entweder den Erwartungen des Akteurs entspricht oder aber als valabler Einstieg in einen Aufstiegskanal taxiert wird. Die Anstellungswechsel in der Versuchsphase folgen einer Trial-and-error-Logik. Weil die Fachhochschulabgänger auf individuellen Pfaden aus ihrem angestammten Milieu ausgebrochen sind, und sie in einer ersten Phase nur selten beratende Vorbilder über das weite wirtschaftliche Feld führen, entscheiden sie sich zuerst für einen Job, der sie ausreichend interessiert und testen darauf, ob dieser ihren Wünschen und Erwartungen entspricht. Mit etwas Glück sind sie rasch an ihrem Ziel. Im gegenteiligen Fall kann es zu einer längeren Suche mit vielen Firmen- und Stellenwechseln kommen. Ein grosser Teil der Befragten kann retrospektiv klar angeben, wann diese Phase für sie geendet hat. Konkret äussert sich dieses Schlusssignal allerdings recht variabel. Es kann die Gründung eines eigenen Unternehmens sein, ein richtungweisender Branchenwechsel oder der Antritt einer Stelle, die längerfristig Sicherheit und Aufstiegschancen verheisst.

Die Versuchsphase gilt dem Entwickeln und Differenzieren sozialer und beruflicher Identität. Dieser Suchprozess ist notwendig für die Aufstiegskandidaten - nur selten nämlich, so können wir aus den Narrationen schliessen, entspricht eine Stellung exakt ihren Träumen. Die Diskrepanz zwischen Wunsch und Wirklichkeit ist beruflichen Aufstiegen eingeschrieben und dient als Brennstoff weiteren Strebens. Der Suchprozess erlaubt den Akteuren ihre Interessen zu entdecken, ihre Stärken und Schwächen zu bewerten und nicht zuletzt die Vorstellung davon zu schärfen, was sie eigentlich anstreben. Die Position und Identität, die sie gegen Ende dieses Prozesses finden, geben den Ausschlag für die nachfolgende Aufstiegsund Konsolidierungsphase. Sie dient als Karriereanker. Laut dem amerikanischen Managementtheoretiker Schein $(1977,52)$ resultiert der Karriereanker «from an interaction between the person with his needs and talents and the work environment with its opportunities and constraints». Als kulturelle Motive, die von den Karriereaspiranten auf ihrer Suche immer wieder neu interpretiert oder geschaffenen werden, spiegeln diese Anker tiefer liegende biographische Dispositionen und deren Konfrontation mit der beruflichen Welt. Was kann man sich nun konkret unter einem solchen Anker vorstellen?

$\mathrm{Zu}$ Beginn der Versuchsphase stehen die Ingenieure und Betriebswirtschafter vor der Wahl zwischen grossen und kleinen Firmen. Mit den folgenden Worten erklärt ein Ingenieur, wie er im Aufeinandertreffen seiner Wünsche mit den Strukturen der Arbeitswelt erkannte, dass er ein "Gewerblertyp» und weniger ein «Industrietyp» sei:

Nach dieser Stelle bei diesem Planungsbüro bin ich ein Jahr lang, bei der Firma X, in der Konstruktion und Berechnung von Hochspannungsapparaten gewesen. Da habe ich noch die industrielle Seite kennengelernt nach dem Studium. Ich habe dann aber feststellen müssen "ich bin nicht der Industriemensch", ich bin irgendwie einfach der "Gwerblermensch". 
Ich habe dann nach einem Jahr bei der Firma X wieder gewechselt und habe dann aber sehr konkret, sehr konkrete Vorstellungen gehabt was ich eigentlich will. (Ingenieur, ${ }^{*} 1957$ )

Der Ausschnitt veranschaulicht, wie der befragte Ingenieur verschiedene Jobs und Positionen im Trial-and-error-Verfahren prüft. Er hat seine Lehre in der Welt der kleinen Unternehmen absolviert, will aber danach die industrielle Welt kennenlernen, nicht zuletzt weil er hofft - wie er zum jetzigen Zeitpunkt etwas verschämt gesteht - dort verheissungsvollere Karrierechancen vorzufinden. Mit dem Alltag des Grossunternehmens konfrontiert, gewinnt er aber schnell den Eindruck, dass seine «Persönlichkeit» nicht dorthin passt. In der Folge wird die Abgrenzung von Gewerbe und Industrie für ihn zum Anker seiner Identitätsbildung und das Gewerbe schliesslich der Sektor, in dem er sich fortentwickeln möchte. Die Welt der kleinen und unabhängigen Firmen fasziniert einen beträchtlichen Anteil der Ingenieure und Betriebswirtschafter. Der Einzelne habe mehr Einfluss, die Wertschöpfung sei sichtbar und könne praktisch in ihrer ganzen Breite mitverfolgt werden; ausserdem erlaube es dieses Milieu den Formalismus der Grossunternehmen zu vermeiden. Genau diese Grossunternehmen andererseits gelten bei den Befragten als reich mit Karrierechancen ausgestattet, um die Aus- und Weiterbildung ihrer Angestellten bemüht und grosszügig, wenn es um die langfristige Karrieresicherheit geht. Gerade weil diese zwei Universen von den Ingenieuren und Betriebswirtschaftern als Gegensätze begriffen werden, erstaunt es nicht, dass im Anschluss an die Versuchsphase nur noch wenige zwischen den beiden Unternehmenstypen wechseln.

Die jungen Ingenieure nehmen ihre Karriere in der Regel in einer Forschungs- oder Entwicklungseinheit eines industriellen Grossunternehmens oder in einem kleinen Planungsbüro in Angriff. In ihren Antworten bezeichnen die Befragten diesen Einstieg als «normal» oder «klassisch» - eine Karrierepassage, die ein Ingenieur notwendigerweise zu durchschreiten hat. Anschliessend stellt sich für die Karriereaspiranten aber rasch die Frage, ob man sich als "Bastler», «Forscher» oder «Techniker» fühlt oder ob man eher dem Verkauf, dem Marketing oder dem Management zugeneigt ist. Im ersten Fall wird die fachliche Kompetenz und eine damit verbundene Denkhaltung schnell zu einem zentralen Karriereanker, der den weiteren Karriereradius auf Positionen wie technischer Teamleader oder Leiter Qualitätskontrolle einschränkt. Die zügige Passung zwischen dem strukturellen Angebot der Industriefirmen und der Identifikation als Bastler führt zu einer eher kurzen und reibungslosen Versuchsphase. Die Ingenieure, die realisieren, dass ihre Ambitionen nicht auf die strukturell verfügbaren Angebote zugeschnitten sind, beginnen nach Alternativen Ausschau zu halten. Es kann für sie darum eine längere und komplizierte Versuchsphase folgen. Die Betroffenen müssen ihre Berufsidentität anpassen. Es gelingt diesen Ingenieuren selten, ganz aus dem industriellen Sektor auszubrechen, meist visieren sie Positionen im Personal-, Verkaufs- oder Marketingbereich innerhalb der Industrie an. Vor allem die technischen Ingenieure beurteilen diese sich 
vom Technischen entfernenden Schritte als riskant, zumal sie den Übergang vom technischen Bereich ins Management im Allgemeinen als unumkehrbar begreifen. Unabhängig davon, ob diese Vorstellungen zur Chronologie zutreffen, wird der Wechsel von technischen Aufgaben zu Stabsaufgaben oder ins Management von Ingenieuren als die zentrale Karriereentscheidung stilisiert. Sie habe weitreichende Folgen für ihre berufliche und soziale Identität.

Die Auswahl potentieller Einstiegspforten ist für Betriebswirtschafter vielfältiger als für die Ingenieure. Die Firmen offerieren einerseits Stellen für BuchhaltungsMarketing- oder Verkaufsspezialisten; zum anderen stehen auch spezifische Einstiegspositionen zur Verfügung: etwa Controllingstellen in Buchprüfungsfirmen, die einen weiten Einblick in verschiedene Wirtschaftsfelder gewähren, «Traineeships» in Grossfirmen, in denen per Rotationsprinzip unterschiedliche Unternehmensgebiete gestreift werden oder aber Assistentenjobs, bei denen man angeleitet von einem höheren Manager einen breiten Einblick in die Firma gewinnt. Gerade wegen dieser Unübersichtlichkeit scheint die Wahl für die Betriebswirtschafter anspruchsvoller und folgenreicher zu sein. Jede dieser Positionen verheisst ein spezifisches Potential und ist mit dementsprechenden Erwartungen aufgeladen. Abgesehen von jenen, die umgehend zu Spezialisten im Marketing oder in der Buchhaltung werden, versuchen die Betriebswirtschafter in dieser Phase "generalistisch» zu bleiben. Sie wollten eine all zu rasche und all zu weitreichende Spezialisierung vermeiden, weil diese zum Karrierehindernis werden könnte, meinten insbesondere jene, die sich gerade in dieser Phase befinden. Diese Vermeidung des Absinkens ins Spezialistentum scheint ihrer Karriere in der Versuchsphase Form und Rhythmus zu geben. Häufige Stellenwechsel, wie sie für die Versuchsphase typisch sind, dienen nicht nur dazu, eine professionelle Identität zu finden. Sie erlauben es, den Karriereaspiranten eine möglichst grosse Anzahl von Aufgaben und Bereichen kennen zu lernen. So erwerben sie eine allgemeine und breite Managementerfahrung, die in ihren Augen ihre Aufstiegschancen erhöht. Während Einstiegsjobs als Trainees oder Direktionsassistenten als offizielle Komponente der Nachwuchsförderung ganz gezielt solche breiten Einblicke bieten, gelten spezialisierte Stellenprofile, insbesondere solche in den weiblich konnotieren Bereichen wie Marketing oder Personal als riskant, weil man von ihnen nicht mehr loskomme. Die als rational und männlich konnotierten, buchhaltungsnahen Fachbereiche geniessen einen Sonderstatus und gelten, vorausgesetzt die technischen Aspekte nehmen nicht überhand, als Sprungbretter für höhere Managementpositionen.

\subsection{Aufstieg und Konsolidierung}

Die Aufstiegs- und Konsolidierungsphase setzt ein, wenn die Ingenieure und Betriebswirtschafter eine Anstellung gefunden zu haben, deren Zukunftsverheissungen mit ihren eigenen Zukunftsvorstellungen zusammenfallen. In den meisten Fällen handelt es sich um den Einstieg in einen Kanal, der einen regelmässigen hierarchi- 
schen Aufstieg oder zumindest eine als logisch empfundene, längerfristig angelegte Abfolge von interessanten Aufgaben verspricht. In gewissen Fällen entspricht dieses imaginierte Sprungbrett einer Firma (zum Beispiel einer für ihre interne Beförderungspolitik bekannten Bank), in anderen einer Funktion (beispielsweise einer Buchhaltungsfunktion, deren Kompetenzen zu einem Aufstieg prädestinieren) oder einer bestimmten Branche (zum Beispiel der Uhrenbranche). Vielfach erkennen die Akteure das Potential einer Position erst nach ein paar Monaten in der Anstellung, der Firma oder der Branche. Selten ist der richtige Einstieg die Folge einer bewussten Entscheidung - eher entpuppt sich eine Stelle nachträglich als das unbewusst Gesuchte. Die Dauer der Aufstiegsphase hängt davon ab, wie lange die Akteure einen weiteren Aufstieg für realistisch halten und wie lange sie deshalb, im Denken und Handeln danach streben. Für ausserordentlich Erfolgreiche kann diese Phase bis in den Karrierenherbst hinein andauern. Die Mehrheit wechselt aber davor in eine Phase, die ich die Konsolidierungsphase nenne: Anstatt weitere Aufstiege anzustreben versuchen die Akteure in dieser Phase ihre Position und die damit verbundenen Privilegien zu verwalten.

Die meisten Ingenieure und Betriebswirtschafter beschreiben ihren Aufstieg als ein Emporklettern auf einer organisationsinternen Karriereleiter. Dafür sei es notwendig, ihre Ambitionen ihren Vorgesetzten zu zeigen, die Verschiebungen im internen Arbeitsmarkt zu beobachten und die Angebote zu packen, die sich ihnen böten. Hierarchieleitern verstehen sie aber durchaus auch als mehrdimensional: Nebst den formalen Positionen spielt zum Beispiel auch die Internationalität einer Aufgabe eine Rolle. Je «internationaler» eine Position, desto höher wird sie von den Befragten eingestuft. Ein zügiges Vorwärtskommen entlang solcher Hierarchien ist für viele gleichbedeutend mit Erfolg. Einer der in diesem Sinne erfolgreicheren Betriebswirtschafter schildert seine Aufstiegsphase folgendermassen:

\section{Ich zähle schnell auf: ich bin Regionalcontroller gewesen, dann bin ich Leiter Systeme gewesen, dann habe ich die Funktion übernommen Controlling Operations, Business Area Operations, dann Controlling Overseas, dann Controlling Europa, dann Controlling Wealth Manage- ment International, dann Controlling Global Wealth Management and Businessbanking, das ist die höchste Controlling-Funktion. Wenn sie das anschauen in diesen 10 Jahren eine klassische, logische Entwicklung und heute in dieser Position die ich heute habe bin ich verantwortlich für 300 Controller weltweit. (Betriebswirtschafter, 1963)}

Der Befragte betrachtet seine Aufstiegsphase in Kategorien wie «erfolgreich», «logisch», nennt sie an anderer Stelle «sauber» oder gar "perfekt». Sein Aufstieg folgt einem schnellen Rhythmus von linearen und zugleich inkrementalen Wechseln, die in hierarchisch immer höhere Sphären und immer internationaleren Gefilde führen. Gegenüber einer solchen ausserordentlich erfolgreichen Karriere fällt das Gros der 
Berufsverläufe ab: sie verlaufen langsamer, bestehen aus weniger Stationen und sind nicht notwendigerweise international.

Eine Minderheit der Befragten, die insbesondere in Unternehmen zu finden sind, in denen sich die Hierarchiestufen zunehmend verwischen, gibt an, aufzusteigen, indem sie im selben Job verbleiben. Ihr Stellenprofil habe sich im Laufe der Zeit stark verändert und dadurch einen höheren Status erhalten, so ihre Argumentation:

Die technischen Rechnungswesen-Aufgaben sind kleiner geworden, ITAufgaben sind gewachsen und jetzt bin ich im IT gelandet ... das hat zwei-, dreimal bewusste Entscheidungen drin gehabt von meiner Seite. Will ich da bleiben? Ich sitze sozusagen auf dem gleichen Stubl aber die Arbeit ist an mir vorbeigezogen ... (Betriebswirtschafter, ${ }^{*} 1971$ )

Insbesondere in Wachstumssektoren der 1990er Jahre, zum Beispiel im Telekommunikations- oder Cateringbereich, fühlen sich die Aufsteiger eher vom Ausbau getragen und mit ihren Jobs nach oben transportiert, als dass sie über eine Sequenz von hierarchisch geordneten Stellen nach oben steigen. Doch auch dieser Aufstieg per Karussell bedarf einer ständigen individuellen Steuerung und Gestaltung. Die Akteure erzählen, sie würden gezielt darauf hinarbeiten, die Relevanz und das Prestige ihres Stellenprofils zu steigern.

Diskussion

Als Ergänzung und Erweiterung quantitativer Analysen sozialer Mobilität bietet es sich an, die interaktionistische Karriereforschung zur Untersuchung von Aufstiegsprozessen zu mobilisieren. Diese verfügt über ein sequentielles Karrierekonzept, vermag das handlungstheoretische Zusammenspiel zwischen sozialen Strukturen und individuellen Motivlagen besser zu fassen und verfügt über einen offeneren, biographisch situierten Repräsentationsbegriff. Um dies zu illustrieren präsentierte ich eine qualitative Studie über Karrieren von Ingenieuren und Betriebswirtschaftern in der Schweiz. Ich rekonstruierte aufsteigende Mobilität als biographischen Prozess, mit seinem Normalitätskontext, seinen vertrackten Zeitstrukturen, seinen Entscheidungsmechanismen und subjektiven Strategien. Diese auf biographischen Interviews beruhende Rekonstruktion sozialen Aufstiegs unterstreicht folgende drei Punkte:

Erstens verändert sich das Streben in Stärke und Ausformung im biographischen Verlauf: Das langsame, vielfach zögerliche Aufflackern des Strebens, die Notwendigkeit der sozialen Bestätigung während der Mauserungsphase, die identitätspolitische Transformation und Konkretisierung in der Versuchsphase, aber auch die Rücknahme der Intensität (wenn das Streben in der Aufstiegsphase «strukturell» nicht erwidert wird) weisen eindringlich auf die Notwendigkeit hin, Deutungsmuster 
in ihrem Verlauf zu studieren. Allgemein kann die Elastizität, die Unbestimmtheit und die Sprunghaftigkeit des zeitlichen Verlaufs von Aufstiegskarrieren anhand der biographischen Erzählungen nachgezeichnet werden. Zudem zeigt sich, dass sich die Bedeutung einer bestimmten Phase nur aus dem Verlauf der ihr vorausgehenden Phase erschliessen lässt, und dass eine Phase nur über ihren Bezug zu einer erträumten Zukunft verstanden werden kann. Ingenieure können sich in Forscherpositionen mit bescheidenen strukturellen Zukunftschancen zufrieden fühlen - dies allerdings nur wenn sie zuvor existierende Alternativen als "Gewerbler» geprüft haben und überzeugt davon sind, dass der Wechsel weg vom «technischen» biographisch irreversibel und deshalb zu risikoreich sei.

In ganz verschiedenen Momenten des Aufstiegsprozesses kann zweitens gezeigt werden, wie dem sozialen Milieu entsprungene Motivlagen mit Opportunitätsstrukturen zusammenspielen und so spezifische Biographieverläufe kreieren. Die für das Milieu der Handwerker und der kleinen Angestellten typische Vorstellung von «Normalität» bezüglich des Ausbildungsverlaufes verbindet sich mit den Opportunitätsstrukturen des Bildungswesens und führt die Befragten in eine Berufslehre. Die strukturell beschränkten Aufstiegschancen von Handwerkern oder kaufmännischen Angestellten wiederum kollidieren mit deren milieutypischen Ambitionen - es kommt zum Ausbruch über die Fachhochschulen. Die Konfrontation mit dem Arbeitsklima in einer grossen Industrieunternehmung bricht sich an der gefühlten Identität als "Gewerbler» und führt zu einem «Rückzug» in Strukturen, die aufgrund ihrer fehlenden hierarchischen Differenzierung nur noch wenig Chancen auf ein Weiterkommen versprechen.

Drittens erhellt die qualitative Analyse der Aufstiegserzählungen von Ingenieuren und Betriebswirtschaftern die enge Verflechtung von hierarchischem mit identitärem Streben. Das Ertasten sozialer Identitäten - «Gewerbler» werden oder sich als «Forscher» fühlen - ist integraler Bestandteil biographischer Aufstiegsprozesse. Welche Positionen Aufstiegswillige erstreben hängt also davon ab, ob die aktuelle Position mit ihrer - biographisch entstandenen - sozialen Identität zusammenpasst bzw. welche sozialen Identitäten sie erstrebenswerten Positionen subjektiv zuschreiben. Deshalb müssen Karrieretheorien imperativ mit einem biographisch situierten Identitätsbegriff arbeiten.

Barley, Stephen R. 1989. "Careers, Identities, and Institutions: the Legacy of the Chicago School of Sociology.» In Michael B. Arthur, Douglas T. Hall und Barbara S. Lawrence (Hrsg), Handbook of Career Theory. Cambridge: Cambridge University Press.

Baron, James N. und William T. Bielby. 1980. Bringing the Firms Back in: Stratification, and the Organization of Work. American Sociological Review, 45(5): 737-765. 
Becker, Howard und Anselm L. Strauss. 1956. Careers, Personality, and Adult Socialization. American Journal of Sociology, 62: 253-263.

Bergman, Manfred Max, Dominique Joye und Beat Fux. 2002. Social Change, Mobility, and Inequality in Switzerland in the 1990s. Schweizerische Zeitschrift für Soziologie, 28(2): 267-295.

Bertaux, Daniel. 2005. Le récit de vie. Paris: Armand Colin.

Bertaux, Daniel (Hrsg). 1981. Biography and Society. Beverly Hills: Sage.

Blau, Peter und Otis D. Duncan. 1967. The American Occupational Structure. New York: Wiley.

Bühlmann, Felix. 2008. The Corrosion of Career - Occupational Trajectories of Business Economists and Engineers. European Sociological Review, 24(5): 601-615.

Caroll, Glenn R. und Karl Ulrich Mayer. 1986. Job-Shift Patterns in the Federal Republic of Germany: The Effects of Social Class, Industrial Sector, and Organizational Size. American Sociological Review, 51(3): 323-341.

De Coninck, Frédéric und Francis Godard. 1989. L'approche biographique à l'épreuve de l'interprétation. Les formes temporelles de la causalité, Revue Française de Sociologie, 31(1): 23-53.

Demazière, Didier und Claude Dubar. 1997. Analyser les entretiens biographiques. L'exemple des récits d'insertion. Paris: Nathan.

Doeringer, Peter B. und Michael J. Piore. 1971. International Labour Markets and Manpower Analysis. Heath: Lexington Books.

Erikson, Robert und John Goldthorpe. 1992. The Constant Flux: a Study of Class Mobility in Industrial Societies. Oxford: Clarendon Press.

Fischer, Wolfram und Martin Kohli. 1987. «Biographieforschung.» In Wolfgang Voges (Hrsg), Methoden der Biographie- und Lebenslaufforschung. Opladen: Leske + Budrich.

Girod, Roger. 1977. Inégalité inégalités. Analyse de la mobilité sociale. Paris: Presses universitaires de France.

Hughes, Everett C. 1937. Institutional Office and the Person. American Journal of Sociology, 43(3): 404-413.

Joye, Dominique; Manfred Max Berman und Paul S. Lambert. 2003. Intergenerational Educational and Social Mobility in Switzerland. Schweizerische Zeitschrift für Soziologie, 29(2): 263-291.

Joye, Dominique und René Levy. 1994. Vers une analyse de la stratification en Suisse. Examen comparatif de quelques classifications. Schweizerische Zeitschrift für Soziologie, 20(2): 405-437.

Kelle, Udo und Susann Kluge. 1999. Vom Einzelfall zum Typus. Opladen: Leske + Budrich.

König, Mario, Hannes Siegrist und Rudolf Vetterli. 1985. Warten und Aufrücken. Die Angestellten in der Schweiz, 1870 - 1950. Zürich: Chronos.

Levy, René, Dominique Joye, Olivier Guye und Vincent Kaufmann. 1997. Tous égaux? De la stratification aux représentations. Zürich: Seismo.

Levy, René und Christian Suter. 2002. Stratification Research in Switzerland: where are we at? Schweizerische Zeitschrift für Soziologie, 28(2): 181-192.

Lipset, Seymour M. und Reinhard Bendix. 1959. Social Mobility in Industrial Societies. Berkley: University of California Press.

Rosenthal, Gabriele. 1995. Erlebte und erzählte Lebensgeschichte. Frankfurt a.M.: Campus.

Schein, Edgar H. 1977. «Career Anchors and Career Paths: A Panel Study of Management School Graduates.» In JohnVan Maanen (Hrsg), Organizational Careers: Some New Perspectives. London: Wiley.

Schmeiser, Martin. 2000. Intergenerationeller Sozialer Abstieg in Schweizer Akdademikerfamilien: eine Fallanalyse. Schweizerische Zeitschrift für Soziologie, 26(3): 637-662.

Schütze, Fritz. 1983. Biographieforschung und narratives Interview. Neue Praxis, 13: 283-293. 
Spilerman, Seymour. 1977. Careers, Labor Market Structure, and Socioeconomic Achievement. American Journal of Sociology, 83(3): 551-593.

Stamm, Hanspeter, Markus Lamprecht und Rolf Nef. 2003. Soziale Ungleichheit in der Schweiz. Strukturen und Wahrnehmungen. Zürich: Seismo.

Strauss, Anselm L. und Juliet Corbin. 1996. Grounded Theory. Grundlagen qualitativer Sozialforschung. Weinheim: Beltz.

Wohlrab-Sahr, Monika. 1995. «Erfolgreiche Biographie - Biographie als Leistung.» In Wolfram FischerRosenthal und Peter Alheit (Hrsg), Biographien in Deutschland. Soziologische Rekonstruktionen gelebter Gesellschaftsgeschichte. Opladen: Westdeutscher Verlag. 